Tim Sandmann† \& Klaus Preisner ${ }^{*}$

\title{
Religiosität und Fertilität: Eine empirische Untersuchung des Einflusses von Religiosität auf Elternschaft und Kinderzahl
}

\section{Religiousness and fertility: An empirical investigation of the effect of religiousness on parenthood and number of children}

\section{Zusammenfassung:}

Anhand der Daten der Allgemeinen Bevölkerungsumfrage der Sozialwissenschaften von 2012 wird für Ost- und Westdeutschland der Einfluss christlicher Religiosität auf a) die Wahrscheinlichkeit der Elternschaft und b) die Anzahl der Kinder mithilfe von Poisson-Logit-Hurdle-Modellen untersucht. Religiosität wird hierbei anhand des mehrdimensionalen Konzepts der Zentralität nach Huber gemessen. Zunächst wird geprüft, ob Religiosität einen Effekt auf Elternschaft und die realisierte Kinderzahl hat, was nur in Westdeutschland der Fall ist. Schließlich wird untersucht, über welche Mechanismen die vorgefundenen Effekte vermittelt werden, wobei als Mediatoren Kindernutzen, Fertilitätsnormen, Bewertung von Geburtenkontrolle, Geschlechterrollen sowie Eheaffinität berücksichtigt werden. Die Mediationsanalysen für Westdeutschland deuten darauf hin, dass religiöse Personen vor allem aufgrund ihrer stärkeren Präferenz für die Ehe häufiger den Übergang zur Elternschaft vollziehen. Obwohl die Mediatoren die erwarteten Zusammenhänge mit Religiosität und Familiengröße aufweisen, kann die höhere Kinderzahl religiöser Personen kaum mit den verwendeten Mediatoren erklärt werden.

\begin{abstract}
:
The effect of religiosity on a) the likelihood to have children, and b) the number of children is analyzed for East- and West-Germany using the Allgemeine Bevölkerungsumfrage der Sozialwissenschaften 2012 data and poisson-logit hurdle models. Religiosity is measured with Huber and Huber's multidimensional concept of centrality. At first, the effect of religiosity on the likelihood to have children and the number of children is analyzed for East and West Germany - showing that religiosity influences fertility only in West Germany. Finally, mediator variables such as value of children, fertility norms, opinion on birth control, traditional gender roles as well as marriage patterns are used to explain why centrality increases fertility in West Germany. The analyses show that religious persons are more likely to have children, in particular because they more often marry. Although the mediator variables show the expected correlations with religiosity and family size, the mediators hardly explain the greater number of children in religious people.
\end{abstract}

* Anmerkung des Co-Autors: Der gemeinsam verfasste Beitrag ist aus einem Forschungsprojekt von Tim Sandmann (Sandmann 2015) hervorgegangen, welches ich betreut habe. Im Verlauf der Begutachtung des Manuskripts ist der Erstautor Tim Sandmann verstorben. Der Beitrag wurde daraufhin von mir auf der Grundlage der Gutachten überarbeitet und mit Einverständnis der Zeitschrift für Familienforschung/Journal of Family Research zur Publikation gebracht. Ich übernehme daher die alleinige Verantwortung. Die Anerkennung gebührt jedoch Tim Sandmann. 
Schlagwörter: Religiosität, Konfession, Fertilität, Hurdle-Models, Fertilitätsnormen, Nutzen von Kindern, Ehe, Geburtenkontrolle, Geschlechterrollen
Key words: religiosity, fertility, hurdle-models, fertility norms, value of children, marriage, birth control, gender roles

\section{Einleitung}

Familie und Elternschaft galten in Deutschland lange als Selbstverständlichkeit im Lebenslauf. Diese Selbstverständlichkeit wurde jedoch zunehmend in Frage gestellt, was sich in einem Geburtenrückgang und in einem Anstieg der Kinderlosigkeit ab den 1980er Jahren äußerte. Die Veränderungen der Fertilität wurden erst in den 1990er Jahren in der Wissenschaft und schließlich in den 2000er Jahren auch in der Politik thematisiert. Einerseits stand dabei die Frage im Vordergrund, welche Faktoren den Geburtenrückgang bewirken, andererseits, wie Familiengründungen und Geburten weiterer Kinder angeregt werden können. Die demographischen Entwicklungen sollten dabei nicht unabhängig von Entwicklungen im Bereich der Religiosität betrachtet werden. Zum einen fand im Zeitraum des Geburtenrückgangs auch eine Säkularisierung statt, welche sich z.B. in der $\mathrm{Zu}$ nahme Konfessionsloser und im Rückgang an Kirchgängern und Gläubigen zeigt (Lois 2011a; Pollack/Müller 2013). Zum anderen ist aus nationalen und internationalen Studien bekannt, dass religiöse Personen im Schnitt mehr Kinder bekommen als säkulare (siehe z.B. Kröhnert/Klingholz 2010).

Dies wirft die Frage auf, über welche Kanäle sich Religiosität auf das Reproduktionsverhalten auswirkt. In der vorliegenden Arbeit wird daher der Einfluss christlicher Religiosität auf Elternschaft und Kinderzahl anhand der Daten der Allgemeinen Bevölkerungsumfrage der Sozialwissenschaften (ALLBUS) 2012 mithilfe von Poisson-Logit- Hurdle-Modellen untersucht. Da bisher - insbesondere für den deutschsprachigen Raum - nur wenige Studien systematisch die Wirkungsweise von Religiosität analysieren, stehen potenzielle Mediatoren im Fokus der Arbeit. Die Analyse wurde auf Konfessionslose und christliche Glaubensgemeinschaften eingeschränkt, da die sehr kleinen Fallzahlen bei anderen Religionsgemeinschaften wie Islam, Judentum oder Hinduismus keine separaten Analysen zulassen.

Wir gehen zunächst auf den Forschungsstand zum Zusammenhang zwischen Religiosität und Fertilität ein und stellen im zweiten Teil den theoretischen Rahmen der Arbeit vor. Im dritten Teil werden verschiedene potenzielle Mediatoren diskutiert: Kindernutzen, Fertilitätsnormen, Bewertung von Geburtenkontrolle, Geschlechterrollen sowie Eheaffinität und -stabilität. Es folgt die Darstellung des Datensatzes, der Operationalisierungen und der Analysestrategie. Anschließend werden die Befunde vorgestellt und diskutiert. ${ }^{\top}$

\section{Forschungsstand}

Verschiedene Analysen deutscher Querschnittsdaten zeigen, dass Angehörige einer Religion eine höhere Fertilität als Konfessionslose aufweisen, und dass außerdem ein Zusammenhang zwischen verschiedenen Indikatoren der Religiosität und der Kinderzahl besteht

1 Das vorliegende Manuskript basiert in seinen Grundzügen auf einer Qualifikationsarbeit zum Thema „Religiosität und Fertilität“, die einer der Autoren verfasst hat. 
(Arránz Becker et al. 2010; Blume et al. 2006; EKD 2014: 48-49; Fuchs 2009: 355; Hubert 2010, 2015; Ramsel 2011).

Der positive demographische Einfluss von Religion bzw. Religiosität konnte auch in anderen Ländern nachgewiesen werden, z.B. in Österreich (Heineck 2006), den USA (Hayford/Morgan 2008) und Spanien (Adsera 2006a). Ferner zeigen ländervergleichende Studien einen positiven Zusammenhang zwischen Religion bzw. Religiosität und der Kinderzahl sowie anderen fertilitätsbezogenen Variablen wie beispielsweise der idealen Kinderzahl (z.B. Philipov/Berghammer 2007, Frejka/Westhoff 2006, Adsera 2006b).

Wir gehen davon aus, dass die Zugehörigkeit zu einer Religion keinen direkten Effekt auf die Fertilität hat, solange diese nicht auch mit Glaube und einer religiösen Praxis einhergeht. Mit dieser Annahme übereinstimmend, steigt in einer Untersuchung von Brose (2006) der Einfluss der konfessionellen Zugehörigkeit auf die Fertilität mit der Intensität religiöser Partizipation und der religiösen Selbsteinschätzung. Auf der anderen Seite ist es fraglich, ob Religiosität auch außerhalb konfessioneller Zugehörigkeit einen gerichteten positiven Effekt auf die Fertilität entfalten kann (Lehrer 2004).

Die vorgefundenen Zusammenhänge können ferner aufgrund der Verwendung von Querschnittsdaten nicht ohne weiteres als kausale Effekte von Religion bzw. von Religiosität interpretiert werden - denn auch ein umgekehrter Einfluss sowie eine wechselseitige Beeinflussung von Religiosität und Fertilität sind denkbar. Lois (2011b) liefert anhand des Sozio-oekonomischen Panels Hinweise, dass zumindest die Kirchgangshäufigkeit mit der Zahl der Kinder ab fünf Jahren steigt. Berghammer findet in den Daten des Dutch Panel hingegen keinen Effekt der ersten Elternschaft auf die Häufigkeit des Kirchgangs (Berghammer 2012). Allerdings stellte sich die Kirchgangshäufigkeit als starker Prädiktor des zukünftigen Reproduktionsverhaltens heraus.

Zudem muss in Betracht gezogen werden, dass religiöse Personen früher Kinder bekommen oder die Geburtenbiografie früher abschließen könnten als säkulare, was bei Stichproben mit einem hohen Anteil unabgeschlossener Geburtsbiografien zu Scheinunterschieden in der durchschnittlichen Kinderzahl führen kann (Brose 2006: 275f.). Zwar kann bei religiösen Frauen in Deutschland ein späterer Übergang zur Elternschaft beobachtet werden, allerdings bekommen religiöse Frauen - gemessen über Kirchenbesuche bzw. Teilnahme an religiösen Veranstaltungen oder Bedeutung von Religion im Alltag - früher ein zweites Kind (Berghammer/Schuster 2010; Arránz Becker et al. 2010). Ein solcher TimingEffekt schließt jedoch nicht aus, dass religiöse Personen zudem häufiger Eltern sind und dann auch mehr Kindern haben.

Schließlich könnten die Unterschiede bezüglich der Fertilität auf sozioökonomische Unterschiede zurückzuführen sein - der sogenannte „characteristics approach“ bzw. die Kompositionshypothese (McQuillan 2004: 26). In der zitierten empirischen Forschungsliteratur finden sich Unterschiede zwischen religiösen und säkularen Personen jedoch auch nach Berücksichtigung von Alter, Einkommen, Geschlecht und Bildung.

Insgesamt sprechen die bisherigen Befunde dafür, dass Religiosität auch unabhängig von den diskutieren Alternativerklärungen einen positiven Effekt auf Fertilität hat. 


\section{Theoretischer Rahmen}

\subsection{Messmodell der Religiosität}

Gemäß Huber (2003) kann Religiosität als persönliches Konstruktsystem definiert werden, das sich von anderen Systemen durch seinen Bezug auf etwas Letztgültiges abgrenzt. Im Christentum ist dieses „Letztgültige“ ein transzendenter und personaler Gott. Persönliche Konstrukte sind subjektive Theorien zur Antizipation und Bewertung von Ereignissen und Verhaltensweisen. Der Einfluss der Religiosität hängt dabei von zwei Parametern ab: Die Stärke des Einflusses ergibt sich erstens aus der Zentralität des religiösen Konstruktsystems. Mit Zentralität ist der hierarchische Status innerhalb der Menge der Konstruktsysteme eines Menschen gemeint. Je höher der Zentralitätsgrad, desto grösser ist die Bedeutung im Vergleich zu anderen Konstruktsystemen und desto weniger wird ein Konstruktsystem von anderen in seiner Wirksamkeit beschränkt. Zweitens wird die Richtung der Effekte durch konkrete theologische Inhalte, wie z.B. in der katholischen Lehre Verhütungs- und Abtreibungsverbote, bestimmt (Müller 2006). Je nach vertretenen Glaubensinhalten kann eine hohe Zentralität des religiösen Konstruktsystems ganz unterschiedliche Wirkungen auf die Geburtenneigung entfalten. So kann eine zölibatär-asketische Ausprägung von Religiosität (wie z.B. bei den Shakers) auch einen negativen Effekt auf die Zahl der Nachkommen haben. Bei den hier untersuchten christlichen Glaubensgemeinschaften dominieren pronatalistische Inhalte, weshalb ein fertilitätsfördernder Effekt angenommen werden kann. Sowohl in historischen Schriften als auch in zeitgenössischen Publikationen der Kirchen finden sich entsprechende Aussagen zur Bedeutung der Familie und Elternschaft, zu Geburtenkontrolle sowie zu Familienidealen (z.B. EKD 2014; KKK 1997). In Abschnitt 4 werden die pronatalistischen Inhalte näher betrachtet.

\subsection{Entscheidungstheoretisches Modell}

Die Entscheidung für oder gegen ein (weiteres) Kind kann zunächst als eine rein rationale Wahl verstanden werden, bei der eine Abwägung von Kosten und Nutzen der Elternschaft und Kinderlosigkeit vorgenommen wird. In Bezug auf den potenziellen Nutzen von Kindern können dabei drei Dimensionen unterschieden werden: materieller Nutzen (z.B. durch Mitarbeit im elterlichen Haushalt), sozialer Nutzen durch Statusgewinn (z.B. wenn sozialen Normen in Bezug auf die Familiengröße entsprochen wird) und emotionaler Nutzen (Nauck 2001). Dem Nutzen von Kindern stehen direkte Kosten (Versorgung der Kinder, Investitionen in die Ausbildung etc.) und indirekte Kosten (Opportunitätskosten) gegenüber. Letztere hängen davon ab, wie stark Kinder Aktivitäten in anderen Handlungsbereichen (z.B. berufliche Karriere) einschränken (Ehrhardt et al. 2012).

Bei der Modellierung von Reproduktionsverhalten als Ergebnis eines rein rationalen Prozesses handelt es sich jedoch um eine vereinfachende Heuristik. Eine explizite Bestimmung und Verrechnung der Vor- und Nachteile von Kindern scheint aufgrund deren Unbestimmtheit sowie eines fehlenden einheitlichen Maßstabes kaum möglich. Ferner finden generative Entscheidungen unter Unsicherheit statt, da die langfristigen Konsequenzen nur begrenzt vorhersehbar sind (ibd.). Neben rationalen Abwägungen sollten da- 
her auch soziale Normen berücksichtigt werden, welche als „Richtwerte“ eine Entscheidungssituation vereinfachen können. Aus dieser Perspektive heraus können folgende Kanäle für den Einfluss von Religiosität auf die Fertilität angenommen werden:

- Verschiebung der wahrgenommenen Kosten-Nutzen-Relation zugunsten einer Entscheidung für (weitere) Kinder

- Vermittlung sozialer Normen in Bezug auf Familiengröße

- Vermittlung von Normen, die Handlungsalternativen zugunsten von Familie einschränken

Im nächsten Abschnitt werden konkrete Beispiele der dargestellten Kanäle diskutiert.

\subsection{Vermittelnde Mechanismen des Effekts von Religiosität}

Im Folgenden werden fünf potenzielle Mediatoren des Effekts von Religiosität auf die Fertilität betrachtet. In der Analyse des Effekts von Religiosität ist die Unterscheidung von zwei möglichen Auswirkungen sinnvoll: Eine hohe Religiosität kann zum einen bewirken, dass Personen sich häufiger für Kinder entscheiden bzw. seltener kinderlos bleiben. Zum anderen ist denkbar, dass religiöse Personen sich durchschnittlich für mehr Kinder entscheiden bzw. mehr Kinder haben. Beide Auswirkungen werden vermutlich über teilweise unterschiedliche Mediatoren vermittelt.

Kindernutzen: In den christlichen Kirchen Deutschlands wird der Wert von Kindern und Familie betont. Beispielsweise werden im Katechismus der katholischen Kirche Kinder als Geschenk Gottes und Kinderreichtum als Zeichen des göttlichen Segens angesehen (KKK 1997: Absatz 2373-2378). Aufgrund des pronatalistischen Narratives dürften religiöse Personen bei sonst gleichen Eigenschaften Kindern einen höheren Nutzen zusprechen. Wie weiter oben bereits erwähnt wurde, kann hierbei zwischen emotionalem, sozialem und materiellem Nutzen unterschieden werden. In der Untersuchung von Brose (2006) weisen konfessionell gebundene Personen Kindern sowohl einen höheren emotionalen als auch einen höheren materiellen Nutzen (z.B. eine erwartete Unterstützung im Alter) zu. Durch die stärkere emotionale Nutzenbewertung von Kindern sollten sich religiöse Personen zwar häufiger für eine Elternschaft entscheiden, nicht aber zwangsläufig für mehr Kinder (Nauck 2001). Viele Kinder sind allerdings dann eine sinnvolle Strategie, wenn zusätzlich auch ein hoher materieller oder sozialer Nutzen, z.B. ein Anerkennungsgewinn durch eine große Kinderzahl, erwartet wird.

Fertilitätsnormen: Die Herkunftsfamilie, das aktuelle soziale Umfeld sowie kirchliche Lehren vermitteln normative Vorstellungen über die ideale, wünschenswerte Familiengröße, wobei religiöse Personen den empirischen Befunden nach eine höhere Kinderzahl für ideal halten und anstreben (Adsera 2006b; Fuchs 2009; Hayford/Morgan 2008, Philipov/Berghammer 2007). Die Befunde können als Hinweise gelesen werden, dass in einem religiösen Umfeld große Familien positiver bewertet werden und Kinderreichtum somit eher mit einem Anerkennungsgewinn gekoppelt ist als in einem säkularen Umfeld, was den sozialen Nutzen von Kindern erhöht. Außerdem könnte eine hohe Kinderzahl auch als „normaler“ gelten und bei der komplexen Entscheidung für oder gegen ein (weiteres) Kind als normativer Richtwert herangezogen werden. Die höhere als Ideal betrachtete 
Kinderzahl religiöser Personen sollte sich vor allem auf die realisierte Kinderzahl und weniger aber auf die Wahrscheinlichkeit überhaupt Kinder zu bekommen auswirken.

Ablehnung der Geburtenkontrolle: Die Verhütung mit Kondomen oder der Pille gilt im Katholizismus als verwerflicher Eingriff in den natürlichen Ablauf der Fortpflanzung (KKK 1997: Absatz 2370). Die Abtreibung wird ebenfalls abgelehnt (KKK 1997: Absatz 2270). Innerhalb des Protestantismus finden sich hingegen unterschiedliche Positionen (Srikanthan/Reid 2008: 130-131). Aus der Sicht der Evangelischen Kirche in Deutschland (EKD) liegt Familienplanung im Verantwortungsbereich des Einzelnen, wobei die Verhütung mittels Kontrazeptiva ausdrücklich befürwortet wird. Abtreibungen werden in der EKD zumindest als letzte Option in bestimmten Situationen respektiert (EKD 2004). Evangelikale Christen, die sowohl in Freikirchen als auch innerhalb der EKD organisiert sind, vertreten in Bezug auf den Abbruch von Schwangerschaften aber auch deutlich konservativere Positionen (Guske 2014:122ff.).

Da die Anwendung „,künstlicher“ Verhütung selbst unter Katholiken (entgegen der offiziellen Sexualmoral der Kirche) nur von einer Minderheit abgelehnt wird (DBK 2014; BDKJ 2014) und zudem die Einstellung zur Verhütung im ALLBUS nicht abgefragt wurde, konzentrierten wir uns im Folgenden auf den Schwangerschaftsabbruch. Verschiedene Befunde zeigen, dass religiöse Menschen den Abbruch einer Schwangerschaft insgesamt stärker ablehnen (Fowid 2005; Pollack/Müller 2013: 24f.; Roloff 2004: 39-43). Zumindest ein Teil des demographischen Effekts von Religiosität auf Fertilität könnte darauf zurückgeführt werden, dass religiöse Personen ungewollte oder ungeplante Schwangerschaften häufiger austragen, da ein Abbruch der Schwangerschaft aus normativen Gründen als Handlungsoption nicht zur Verfügung steht.

Geschlechterrollen: Die bisherige Forschung zeigt, dass religiöse Personen stärker als säkulare Personen an traditionellen Geschlechterrollen festhalten, welche Frauen eine Rolle als Hausfrau und Kinderbetreuerin und Männern eine Rolle als Versorger der Familie zuweisen (Becher/El-Menouar 2014; Diel/König 2011; Fuchs 2009: 356f.; Fuchs 2014: 122; Wenzel 2010: 70). Traditionelle Geschlechterrollen schränken die biographischen Optionen von Frauen durch die Festlegung auf die Kindererziehung ein und reduzieren hierdurch die Opportunitätskosten von Kindern. Sind traditionelle Geschlechterrollen Bestandteil des Bildes eines „guten christlichen Lebens“, kann ferner angenommen werden, dass diesem Bild zu entsprechen als befriedigend wahrgenommen und von der Gemeinschaft mit Anerkennung und Unterstützung honoriert wird (Lehrer 2004). Traditionelle Geschlechterrollen sollten demnach eine Elternschaft bzw. weitere Kinder und den damit häufiger einhergehenden Verzicht auf eine berufliche Karriere attraktiver machen.

Eheaffinität: Wird die innerfamiliäre Arbeitsteilung an traditionellen Geschlechterrollen ausgerichtet und fehlen gleichzeitig familienexterne Betreuungseinrichtungen, sind Frauen oftmals gezwungen, zeitweilig auf eine Erwerbstätigkeit zu verzichten, wenn ein bestehender Kinderwunsch realisiert werden soll (Wenzel 2010: 59f.). Ein solcher Verzicht führt Frauen aber in die ökonomische Abhängigkeit vom Mann und birgt das Risiko eines Wohlfahrtsverlustes im Falle einer Trennung. Unter diesen Bedingungen hat die Institution der Ehe gegenüber nichtehelichen Lebensgemeinschaften Vorteile: Es wird eine längerfristige Beziehung in Aussicht gestellt und im Scheidungsfall gibt es eine umfassendere ökonomische Absicherung. Hierdurch werden die Risiken der Spezialisierung auf Kin- 
der und Hausarbeit für Frauen reduziert, was die Entscheidung für ein erstes Kind bzw. weitere Kinder begünstigten sollte (Brose 2006: 263f.; Esser 2002: 32).

Religiöse Personen heiraten häufiger und weisen ein geringeres Scheidungsrisiko auf (Kraft/Neimann 2009; Lois 2008; Wagner/Weiß 2003). Demnach sollte der Einfluss von Religiosität auf die Realisierung eines Kinderwunsches sowie eine höhere Kinderanzahl teilweise durch die größere Eheaffinität bei religiösen Personen erklärt werden können.

\section{Methode}

\subsection{Datensatz und Operationalisierung}

Die Überprüfung der Hypothesen erfolgt mithilfe der Daten der ALLBUS-Erhebung von 2012. Jede ALLBUS-Welle hat thematische Schwerpunkte, welche im 10-Jahres-Abstand erneut abgefragt werden. 2012 bildete „Religion und Weltanschauung“ den Themenschwerpunkt der Erhebung. Außerdem ist das Modul „Familie und sich ändernde Geschlechtsrollen" des International Social Survey Programme (ISSP) in die Befragung integriert. Damit gewährleisten die Daten sowohl eine differenzierte Messung von Religiosität anhand des Modells der Zentralität von Huber als auch eine umfassende Analyse der vermittelnden Mechanismen des Effekts von Religiosität auf Fertilität. Huber (2003) schlägt als Proxy zur Messung von Zentralität die Häufigkeit und Intensität der Aktivierung des religiösen Konstruktsystems vor. Er greift hierbei auf das Messmodell von Glock zurück und geht davon aus, dass die Religiosität eines Menschen in fünf primären Dimensionen zum Ausdruck kommt: Ideologie, öffentliche und private Praxis, Erfahrung sowie kognitives Interesse. Jeder der fünf Dimensionen wurde eine Frage des ALLBUS 2012 zugeordnet.

- Ideologie: Zustimmung zur Aussage „Es gibt einen Gott, der Gott für uns sein will.“ (1=,,Stimme voll und ganz zu“ - 5=,,Stimme überhaupt nicht zu“)

- öffentliche Praxis: Kirchgangshäufigkeit (1=,Mehr als Einmal in der Woche“ $\left.6=, \mathrm{Nie}^{\prime \prime}\right)$

- private Praxis: Gebetshäufigkeit (1=,,Täglich“ - 7=,„Nie“) $)^{2}$

- Erfahrung: Häufigkeit der Erfahrung der Nähe Gottes (1=,,Sehr oft“ - 5=,,Nie“)

- kognitives Interesse: Häufigkeit der Beschäftigung mit Glaubensfragen $(1=$, ,Sehr oft“ $\left.-5=, \mathrm{Nie}^{\prime \prime}\right)$

Die Dimensionalität der fünf Items wurde mit einer Hauptkomponentenanalyse untersucht (Cronbachs alpha 0.88). ${ }^{3}$ Die Ergebnisse der Analyse sprechen für die Extraktion einer Komponente (Eigenwert 3.42, Varianzaufklärung 0.69), wobei die einzelnen Items allesamt sehr hohe Werte bei den Eigenvektoren $(>0.79)$ aufweisen. Um die Interpretation zu

2 In der Abfrage wurde nicht zwischen privaten Gebeten und Gebeten im Gottesdienst differenziert. Daher kann nicht ausgeschlossen werden, dass die Operationalisierung der privaten Praxis auch Gebete im Gotteshaus - öffentliche Praxis - miteinschließt.

3 Die Zahl der Komponenten wurde mit der Parallelanalyse nach Horn und mit dem Minimum Average Partial Test bestimmt (Dinno 2009; Bühner 2011). Beide Kriterien deuten darauf hin, dass die Interkorrelationen der Items durch eine Komponente beschrieben werden können. 
vereinfachen werden daher anstatt principal component scores die Mittelwerte der Items als allgemeine Zentralitäts-Skala verwendet. ${ }^{4}$ Zudem wird die Religionszugehörigkeit berücksichtigt, wobei zwischen christlich-konfessionellen und Personen ohne Zugehörigkeit zu einer Religionsgemeinschaft unterschieden wird. ${ }^{5}$

Die drei Nutzendimensionen von Kindern werden über die Zustimmung zu den Items „Zu beobachten, wie Kinder groß werden, ist die größte Freude im Leben“ (emotionaler Nutzen), „Kinder zu haben, erhöht das Ansehen der Eltern in der Gesellschaft“ (sozialer Nutzen) und „Wenn Eltern alt geworden sind, sind ihre erwachsenen Kinder eine wichtige Hilfe für sie" (materieller Nutzen) aus dem ISSP-Modul gemessen, wobei die Items nur schwach korrelieren $(\leq 0.2)$. Fertilitätsnormen werden durch die, im gleichen Modul abgefragte, ideale Kinderzahl operationalisiert. Die Bewertung des Abbruchs einer Schwangerschaft, wenn eine Frau kein weiteres Kind mehr möchte, wird als Indikator für die Einstellung gegenüber Abtreibungen herangezogen. Als Proxy der Präferenz für die Ehe und damit eine stabile Partnerschaft dient der aktuelle Familienstand. Die ursprüngliche Variable wurde hierbei Dummy-kodiert, mit der Ausprägung „ledig/geschieden/getrennt lebend" als Referenzkategorie und eigenständigen Dummy-Variablen für verheiratete und verwitwete Fälle. Eingetragene Lebenspartnerschaften wurden aufgrund der sehr geringen Fallzahl sowie der in Hinblick auf Fertilität anderen Gegebenheiten von der Analyse ausgeschlossen. Zur Operationalisierung der Einstellung gegenüber traditionellen Geschlechterrollen werden sieben Items aus dem ISSP-Modul verwendet, bei denen es vor allem um die Bewertung weiblicher Erwerbstätigkeit geht. Die Items wurden ebenfalls einer Hauptkomponentenanalyse unterzogen. Zwei der sieben Items werden aufgrund der sehr niedrigen Werte bei den Eigenvektoren $(<0.3)$ von der weiteren Analyse ausgeschlossen. Von den verbliebenen Items wird wiederum der Mittelwert gebildet (Cronbachs alpha 0.84). ${ }^{6}$

\subsection{Datenanalyse}

Die Datenanalyse erfolgt mithilfe von Poisson-Logit-Hurdle-Modellen, welche aus zwei Gleichungen bestehen (Hilbe 2014). Mit der ersten Gleichung, einer binären logistischen Regression, wird ermittelt, welchen Einfluss die unabhängigen Variablen darauf haben, ob eine Person Kinder hat oder nicht. Für Personen mit Kind(ern) wird im zweiten Teil

4 Die Items werden hierfür auf einen einheitlichen Wertebereich gebracht (siehe hierzu Huber/Huber 2012: 720). Wenn auf einem der Items ein Wert fehlt, wird der Fall von der Mittelwertbildung ausgeschlossen.

5 Zwischen den christlichen Konfessionen bestanden in der Regel keine signifikanten Unterschiede, weshalb diese zusammengefasst wurden.

6 Bei den Items handelt es sich um die Zustimmung zu folgenden Aussagen:

„Ein Kind, das noch nicht zur Schule geht, wird wahrscheinlich darunter leiden, wenn seine Mutter berufstätig ist.“

„Alles in allem: Das Familienleben leidet darunter, wenn die Frau voll berufstätig ist.“

„Einen Beruf zu haben ist ja ganz schön, aber das, was die meisten Frauen wirklich wollen, sind ein Heim und Kinder.“

„Hausfrau zu sein ist genauso erfüllend wie gegen Bezahlung zu arbeiten.“

„Die Aufgabe des Mannes ist es, Geld zu verdienen, die der Frau, sich um Haushalt und Familie zu kümmern.“ 
mit einer Truncated-at-Zero-Poisson-Regression der Zusammenhang zwischen den unabhängigen Variablen und der Kinderzahl geschätzt. In beiden Modellteilen werden im Folgenden Mediationsanalysen eingesetzt, um die vermittelnden Mechanismen des Effekts von Zentralität zu identifizieren. Hurdle-Modelle haben gegenüber einer herkömmlichen Poisson-Regression im vorliegenden Fall zwei wichtige Vorteile: Zum einen können die statistischen Effekte der Zentralität und der Mediatoren auf die Elternschaft getrennt vom Einfluss auf die Familiengröße untersucht werden. Zum anderen kann mit einem HurdleModell bzw. einer Truncated-at-zero-Poisson-Gleichung das Problem abgefangen werden, dass in den ALLBUS-Daten mehr Kinderlose (=Nullen) zu finden sind, als unter der Poisson-Verteilung zu erwarten wären.

Personen mit nicht-christlicher Konfession $(\mathrm{n}=110)$ wurden aus der Datenanalyse ausgeschlossen. In einigen anderen Studien wurde über eine Alterseingrenzung (z.B. 3545jährige bei Blume et al. 2006) die Stichprobe auf Personen mit weitestgehend abgeschlossener Geburtenbiografie beschränkt. Auf eine solche generelle Eingrenzung haben wir aufgrund der sonst teilweise sehr geringen Fallzahlen verzichtet. Die Berücksichtigung von Personen mit nicht abgeschlossener Geburtenbiografie kann allerdings zu Verzerrungen führen. Bekommen religiöse Personen später ihr erstes Kind (siehe 2.), könnte der Effekt von Religiosität auf die Wahrscheinlichkeit überhaupt Kinder zu haben, nach unten verzerrt werden. Etwaige Verzerrungen des Effekts von Religiosität auf die Kinderzahl von Eltern hängen hingegen von Unterschieden bei der zeitlichen Lage der zweiten und dritten Geburt ab. Als Kontrolle wurden die Analyse an zentralen Stellen auch mit einer eingeschränkten Stichprobe mit ab 45jährigen und damit Personen mit weitestgehend abgeschlossener Geburtenbiografie durchgeführt.

Um einen großen Fallzahlenverlust zu vermeiden, wurde in den Regressionsanalysen bei Personen, die aufgrund des Methodensplits das ISSP-Modul ${ }^{7}$ „Familie und sich ändernde Geschlechtsrollen" nicht beantwortet haben, bei den entsprechenden Variablen (Nutzenvariablen, ideale Kinderzahl, traditionelle Geschlechterrollen) eine einfache Imputation durchgeführt, wobei ein fixer, konstanter Wert eingesetzt wurde. Mit einem Dummy wird in den Regressionsmodellen für die Imputation kontrolliert. Aufgrund der Zufallsauswahl beim Methodensplit kann davon ausgegangen werden, dass keine Systematik (missing completely at random) bei diesen fehlenden Werten vorliegt. Hiermit übereinstimmend zeigte ein Vergleich der beiden Teilstichproben keine systematischen Unterschiede bei den verwendeten Variablen bzw. den Koeffizienten in den Modellen.

\section{Ergebnisse}

Die Ergebnisdarstellung gliedert sich in vier Abschnitte. Im ersten Abschnitt (5.1) wird die Verteilung von Fertilität und Zentralität in der Stichprobe beschrieben. Im zweiten Abschnitt (5.2) werden die diskutieren Mediatoren auf einen Zusammenhang mit Zentralität einerseits und Fertilität andererseits geprüft. Schließlich werden im dritten und vierten Abschnitt die Zusammenhänge zwischen Zentralität und der Chance Kinder zu haben

7 Es gab zwei ISSP-Module, zu denen die Befragten per Zufall zugewiesen wurden. Für ca. die Hälfte der Befragten fehlen daher Werte für einen Teil der Mediatoren. 
(5.3) bzw. der Kinderzahl (5.4) genauer in den Blick genommen. Einerseits wird in diesen Abschnitten geprüft, wie sich Zentralität auf Fertilität auswirkt - auch unter Kontrolle von sozioökonomischen Drittvariablen wie Alter, Einkommen, Bildung und Geschlecht. Anderseits wird untersucht, warum sich Zentralität auf Fertilität auswirkt, was also die vermittelnden Mechanismen für die Zentralitätseffekte sind.

\subsection{Zentralität und Fertilität in Ost- und Westdeutschland: deskriptive Analysen}

Durch die Mittelwertbildung der fünf zur Operationalisierung der Zentralität herangezogenen Items ergibt sich eine Skala, die von 0 (niedrige Zentralität) bis 4 (hohe Zentralität) reicht. Zwischen Ost- und Westdeutschland zeigen sich dabei deutliche Unterschiede (Abbildung 1). Erwartungsgemäß befinden sich Befragte in den neuen Bundesländern mehrheitlich im unteren und Befrage aus den alten Bundesländern im mittleren Segment der Zentralitätsskala.

Abbildung 1: Zentralität in Ost- und Westdeutschland

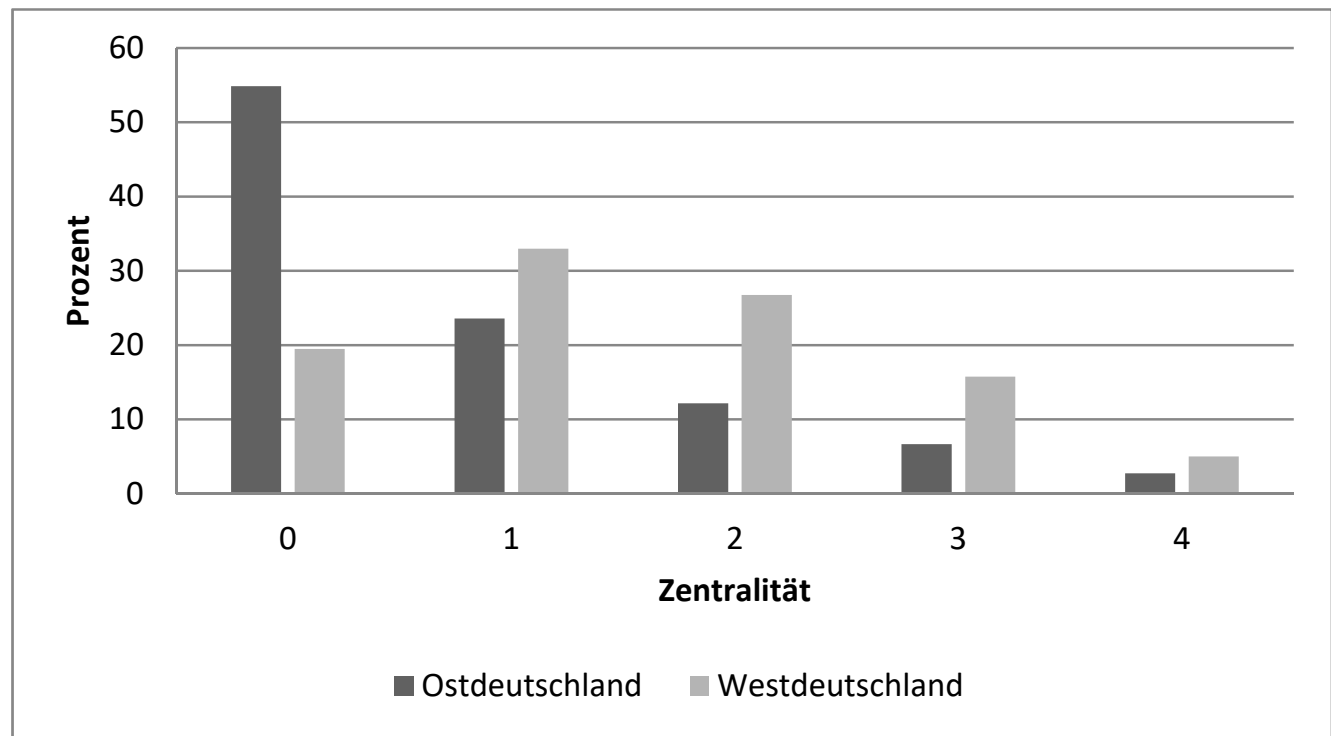

Daten: ALLBUS 2012, eigene Berechnungen, gewichtet, $\mathrm{N}_{\mathrm{Ost}}=1002 / \mathrm{N}_{\text {West }}=2022$.

Die Zentralitätswerte wurden auf ganze Zahlen gerundet.

Erhebliche Unterschiede zwischen Ost- und Westdeutschland zeigen sich auch bei Elternschaft und Kinderzahl (Abbildung 2). In Westdeutschland ist der Anteil kinderloser Befragter mit knapp 39\% deutlich höher als in Ostdeutschland (29\%). Dieser Unterschied ist bereits aus früheren Studien bekannt und könnte z.B. auf den höheren Anteil Konfessionsloser in Ostdeutschland zurückgeführt werden (Arránz Becker et al. 2010: 40; Destatis 2012: 11; Kreyenfeld 2003). 
Abbildung 2: Elternschaft und Kinderzahl in Ost- und Westdeutschland

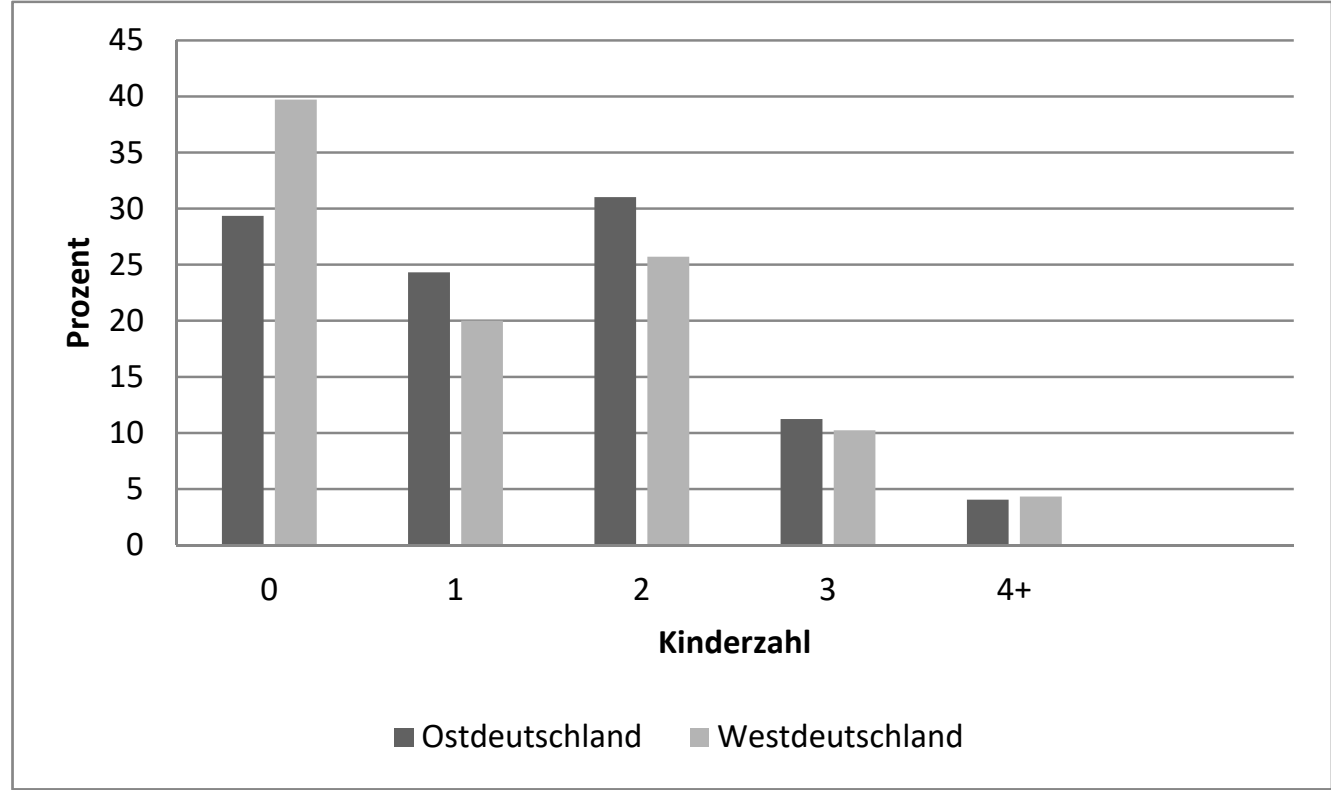

Daten: ALLBUS 2012, eigene Berechnungen, gewichtet, $\mathrm{N}_{\text {Ost }}=1111 / \mathrm{N}_{\text {West }}=2217$.

Abbildung 3: Zentralität und Kinderzahl in Ost- und Westdeutschland

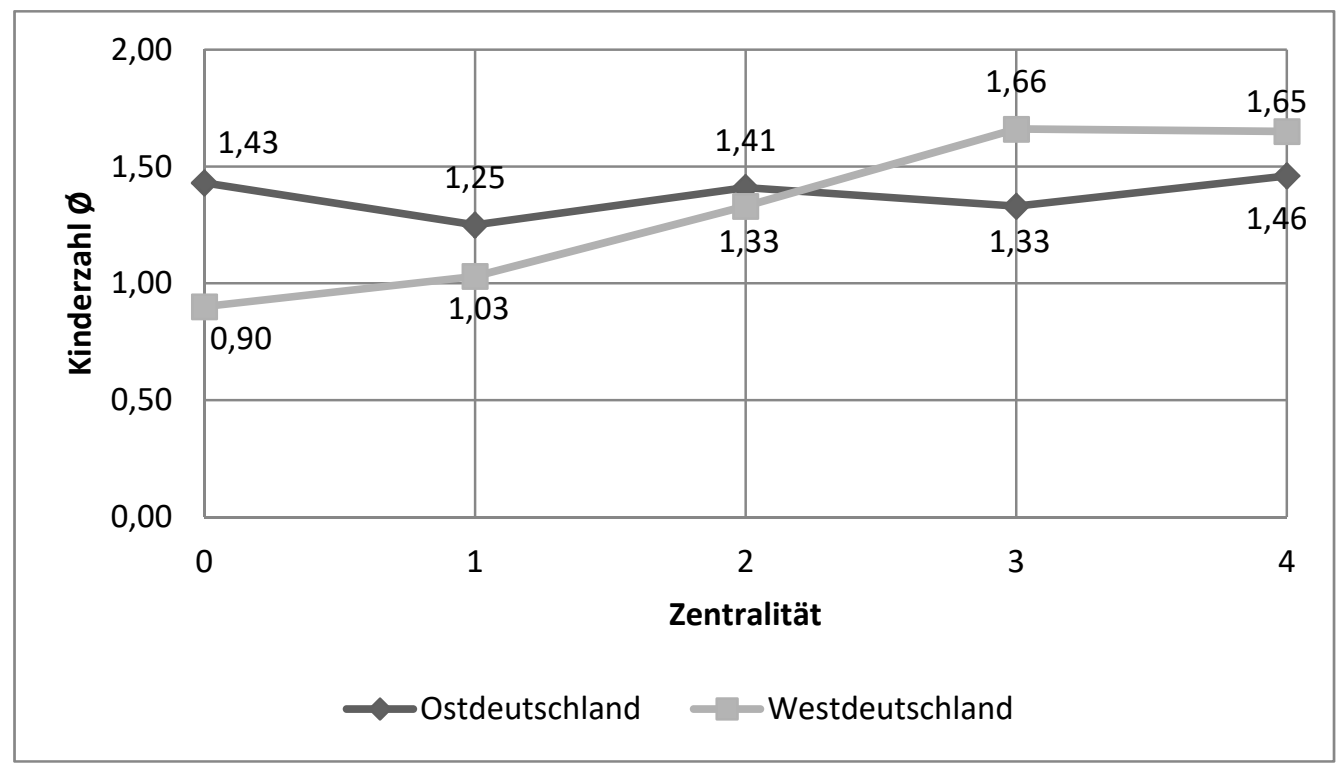

Daten: ALLBUS 2012, eigene Berechnungen, gewichtet, $\mathrm{N}_{\text {Ost }}=999 / \mathrm{N}_{\text {West }}=2001$.

Die Zentralitätswerte wurden auf ganze Zahlen gerundet. 
In Abbildung 3 sind die durchschnittlichen Kinderzahlen für jede Zentralitätskategorie dargestellt. Bereits auf dieser Analyseebene zeigen sich Unterschiede zwischen Ost- und Westdeutschland. Ein stärkerer Zusammenhang zwischen Zentralität und Fertilität scheint nur bei westdeutschen Befragten zu bestehen. Zwischen der höchsten Kategorie der Zentralität und der niedrigsten ergibt sich bezüglich der durchschnittlichen Kinderzahl eine Differenz von 0,75 in den alten Bundesländern - was über 50\% der durchschnittlichen Fertilität entspricht (Punkte-Linie). In den neuen Bundesländern zeigt sich kein (linearer) Zusammenhang zwischen Zentralität und der Kinderanzahl (durchgezogene Linie).

\subsection{Zentralität, Fertilität und potenzielle Mediatoren}

Bei der Untersuchung der vermittelnden Mechanismen zwischen Zentralität und Fertilität interessieren zum einen die Zusammenhänge zwischen Zentralität und den Mediatoren, zum anderen die Zusammenhänge zwischen den Mediatoren und Elternschaft bzw. Kinderzahl. Die Zusammenhänge werden in Regressionsmodellen unter Kontrolle soziodemografischer Variablen für Westdeutschland ermittelt und in Tabelle 1 präsentiert. Auf eine Darstellung für Ostdeutschland wird aufgrund des fehlenden Zusammenhangs zwischen Religiosität und Fertilität verzichtet (siehe nächster Punkt).

Tabelle 1: Zentralität, Fertilität und potentielle Mediatoren (Regressionsanalysen)

\begin{tabular}{|c|c|c|c|}
\hline & $\begin{array}{c}\text { Modelle } 1 \\
\text { Zentraliät } \rightarrow \\
\text { Mediator }\end{array}$ & $\begin{array}{c}\text { Modelle } 2 \\
\text { Mediator } \rightarrow \\
\text { Elternschaft }\end{array}$ & $\begin{array}{c}\text { Modelle } 3 \\
\text { Mediator } \rightarrow \\
\text { Anzahl Kinder }\end{array}$ \\
\hline Zustimmung: Großwerden der Kinder=größte Freude & $0.070^{* *}$ & $0.848^{* * *}$ & $0.139^{*}$ \\
\hline Zustimmung: Kinder erhöhen gesellschaftliches Ansehen & $0.113^{* *}$ & 0.000 & 0.013 \\
\hline Zustimmung: Kinder sind Hilfe im Alter & 0.038 & 0.031 & 0.042 \\
\hline Ideale Kinderzahl & $0.120^{\star * \star}$ & 0.051 & $0.104^{* * *}$ \\
\hline Negative Beurteilung Schwangerschaftsabbruch & $0.356^{* * *}$ & 0.133 & $0.109^{* *}$ \\
\hline Traditionelle Geschlechterrollen & $0.191^{* * *}$ & 0.214 & $0.172^{* * *}$ \\
\hline \multicolumn{4}{|l|}{ Familienstand (Ref.: ledig) } \\
\hline Verheiratet & $0.191^{*}$ & $2.026^{* * *}$ & $0.243^{*}$ \\
\hline Verwitwet & -0.143 & $1.317^{* *}$ & 0.257 \\
\hline $\mathbf{N}$ & 776 & 768 & 530 \\
\hline
\end{tabular}

Daten: ALLBUS 2012, eigene Berechnungen, ungewichtet. Modelle 1: Lineare Regressionen Mediator auf Zentralität (bei Familienstand: multinomiale logistische Regression). Modelle 2: Logistische Regressionen Elternschaft auf Mediator. Modelle 3: Truncated-at-zero-Poisson-Regressionen, Anzahl Kinder (nur Eltern) auf Mediator. Dargestellt werden jeweils unstandardisierte Koeffizienten. Signifikanzniveau: Weitere Variablen in den Modellen: Alter in Jahren (zentriert), Alter ${ }^{2}$, Nettoeinkommen in $€$, Dummy für fehlende Werte beim Nettoeinkommen, Geschlecht, Dummy-Variablen für den höchsten Schulabschluss. ${ }^{*} \mathrm{p}<0.05,{ }^{* *}$ $\mathrm{p}<0.01, * * * \mathrm{p}<0.001$.

Mit einer Ausnahme beeinflusst Zentralität alle Mediatoren in der erwarteten Richtung. Allein die Zustimmung zur Aussage, Kinder sind eine große Hilfe im Alter (materieller Nutzung) steht in keinem signifikanten Zusammenhang mit der Zentralität (Modelle 1).

Augenfällig ist jedoch, dass einige der diskutierten Mediatoren keinen Einfluss auf Elternschaft oder die realisierte Kinderzahl haben. In Hinblick auf Elternschaft (Modelle 2) erweisen sich die Variablen „Aufwachsen der Kinder ist größte Freude“ (emotionaler Nutzen) sowie die Ehe als potenzielle Mediatoren, die genauer in den Blick genommen werden soll- 
ten. Für den Zusammenhang zwischen Zentralität und der Anzahl der Kinder (Modelle 3) zeigen sich signifikante Einflüsse auf die Kinderzahl bei den folgenden Mediatoren: „Aufwachsen der Kinder ist größte Freude“, Ideale Kinderzahl, Beurteilung Schwangerschaftsabbruch, Familienstand und traditionelle Geschlechterrollen. Die beiden Mediatoren „Kinder zu haben erhöht das Ansehen der Eltern in der Gesellschaft“ (sozialer Nutzen) sowie „Wenn Eltern alt geworden sind, sind ihre erwachsenen Kinder eine wichtige Hilfe für sie" (materieller Nutzen) haben weder auf Elternschaft noch auf die Kinderzahl einen signifikanten Einfluss.

Da ein vermittelnder Mechanismus grundsätzlich nur dann vorliegen kann, wenn der Mediator (Z) sowohl mit Zentralität (X) als auch mit Fertilität (Y) zusammenhängt, werden in den folgenden detaillierten Analysen nur noch die potenziellen Mediatoren berücksichtigt, die beide Kriterien erfüllen.

\subsection{Zentralität, Elternschaft und vermittelnde Mechanismen: Multivariate Analysen}

Die Ergebnisse der Poisson-Logit-Hurdle-Modelle werden in zwei Schritten vorgestellt. Tabelle 2 zeigt die Befunde zur Elternschaft bzw. die Ergebnisse der ersten Gleichung (logistische Regression) der Poisson-Logit-Hurdle-Modelle. In den für Ost- und Westdeutschland getrennt durchgeführten Analysen wurde dabei schrittweise vorgegangen. Zunächst wurde die Konfessionalität in das Regressionsmodell aufgenommen (Modelle 4.1 und 7.1) und anschließend um Zentralität ergänzt (Modelle 5.1 und 8.1), so dass klar zwischen den Effekten von Religionszugehörigkeit einerseits und Religiosität andererseits unterschieden werden kann. Ferner wurde geprüft, ob es eine Interaktion zwischen Konfession und Zentralität gibt, sich Religiosität also bei konfessionell gebundenen und konfessionslosen Personen anders auswirkt - dies ist nicht der Fall, weshalb nicht weiter darauf eingegangen wird. In den Modellen 6.1 und 9.1 wird untersucht, inwieweit die vorgefundenen Effekte von Zentralität bestehen bleiben, wenn die sozioökonomischen Merkmale Alter, Nettoeinkommen des Befragten, Geschlecht und höchster Schulabschluss berücksichtigt werden. In den Modellen 10.1 bis 15.1 (nur Westdeutschland) wurden die Mediatoren zunächst einzeln und schließlich zusammen aufgenommen, um die Mechanismen des Zusammenhangs zwischen Zentralität und Fertilität zu identifizieren.

Bei den Analysen in Tabelle 2 fällt zunächst auf, dass Konfessionszugehörigkeit und Zentralität in Ostdeutschland keinen signifikanten Einfluss auf die Wahrscheinlichkeit, Kinder zu haben, ausüben. Aufgrund des fehlenden Zusammenhangs wird entsprechend auf eine detaillierte Mediationsanalyse für die neuen Bundesländer verzichtet. Für Westdeutschland weisen die schrittweise aufgebauten Modelle hingegen einen signifikanten Zusammenhang zwischen Zentralität und dem Übergang zur Elternschaft aus. ${ }^{8}$ Der Effekt von Konfession sinkt hingegen deutlich, wenn die Zentralität kontrolliert wird. Übereinstimmend mit den Erwartungen zeigt sich damit weder ein deutlicher von der Zentralität unabhängiger Effekt von Konfession auf die Elternschaft, noch ein signifikanter Einfluss von Zentralität außerhalb konfessioneller Bindung.

8 Der Unterschied zwischen West- und Ostdeutschland beim Effekt von Zentralität ist signifikant (Ergebnisse nicht dargestellt). 
Tabelle 2: Zentralität und Elternschaft: Mediationsanalyse (Poisson-logit-HurdleRegressionen, 1. Gleichung Logit)

\begin{tabular}{|c|c|c|c|c|c|c|c|c|c|c|c|c|}
\hline & \multicolumn{3}{|c|}{ Ostdeutschland } & \multicolumn{9}{|c|}{ Westdeutschland } \\
\hline & $\begin{array}{c}\text { Modell } \\
4.1 \\
\end{array}$ & $\begin{array}{c}\text { Modell } \\
5.1 \\
\end{array}$ & $\begin{array}{c}\text { Modell } \\
6.1 \\
\end{array}$ & $\begin{array}{c}\text { Modell } \\
7.1 \\
\end{array}$ & $\begin{array}{c}\text { Modell } \\
8.1 \\
\end{array}$ & $\begin{array}{c}\text { Modell } \\
9.1 \\
\end{array}$ & $\begin{array}{c}\text { Modell } \\
10.1 \\
\end{array}$ & $\begin{array}{c}\text { Modell } \\
11.1 \\
\end{array}$ & $\begin{array}{c}\text { Modell } \\
12.1 \\
\end{array}$ & $\begin{array}{c}\text { Modell } \\
13.1 \\
\end{array}$ & $\begin{array}{c}\text { Modell } \\
14.1 \\
\end{array}$ & $\begin{array}{c}\text { Modell } \\
15.1 \\
\end{array}$ \\
\hline $\begin{array}{l}\text { Konfessions- } \\
\text { losigkeit }\end{array}$ & $\begin{array}{r}-0.092 \\
(0.18)\end{array}$ & $\begin{array}{r}-0.197 \\
(0.24)\end{array}$ & $\begin{array}{r}-0.018 \\
(0.30)\end{array}$ & $\begin{array}{c}-0.414^{\star \star} \\
(0.13)\end{array}$ & $\begin{array}{r}-0.075 \\
(0.14)\end{array}$ & $\begin{array}{r}-0.293 \\
(0.17)\end{array}$ & $\begin{array}{c}-0.205 \\
(0.17)\end{array}$ & $\begin{array}{r}-0.292 \\
(0.17)\end{array}$ & $\begin{array}{r}-0.272 \\
(0.17)\end{array}$ & $\begin{array}{r}-0.286 \\
(0.17)\end{array}$ & $\begin{array}{r}-0.030 \\
(0.18)\end{array}$ & $\begin{array}{l}0.051 \\
(0.19)\end{array}$ \\
\hline Zentralität & & $\begin{array}{c}-0.072 \\
(0.11)\end{array}$ & $\begin{array}{c}-0.015 \\
(0.14)\end{array}$ & & $\begin{array}{l}0.318^{\star \star \star} \\
(0.06)\end{array}$ & $\begin{array}{l}0.156^{*} \\
(0.07)\end{array}$ & $\begin{array}{l}0.149^{\star} \\
(0.07)\end{array}$ & $\begin{array}{l}0.157^{*} \\
(0.07)\end{array}$ & $\begin{array}{l}0.133 \\
(0.07)\end{array}$ & $\begin{array}{l}0.150^{\star} \\
(0.07)\end{array}$ & $\begin{array}{l}0.112 \\
(0.07)\end{array}$ & $\begin{array}{l}0.116 \\
(0.08)\end{array}$ \\
\hline $\begin{array}{l}\text { Alter (in } 10 \text { Jahren, } \\
\text { zentriert) }\end{array}$ & & & $\begin{array}{c}0.730^{\star \star *} \\
(0.07)\end{array}$ & & & $\begin{array}{l}0.514^{\star \star \star} \\
(0.04)\end{array}$ & $\begin{array}{l}0.520^{\star * *} \\
(0.04)\end{array}$ & $\begin{array}{l}0.514^{\star \star *} \\
(0.04)\end{array}$ & $\begin{array}{l}0.516^{\star \star \star} \\
(0.04)\end{array}$ & $\begin{array}{c}0.509^{\star \star \star} \\
(0.04)\end{array}$ & $\begin{array}{l}0.322^{\star \star *} \\
(0.05)\end{array}$ & $\begin{array}{l}0.324^{\star \star \star} \\
(0.05)\end{array}$ \\
\hline Alter $^{2}$ & & & $\begin{array}{c}-0.206^{* * *} \\
(0.04)\end{array}$ & & & $\begin{array}{c}-0.219^{\star * *} \\
(0.02)\end{array}$ & $\begin{array}{c}-0.228^{\star \star *} \\
(0.02)\end{array}$ & $\begin{array}{l}-0.220^{\star * *} \\
(0.02)\end{array}$ & $\begin{array}{c}-0.219^{\star \star \star} \\
(0.02)\end{array}$ & $\begin{array}{c}-0.223^{\star \star *} \\
(0.02)\end{array}$ & $\begin{array}{l}-0.188^{* * *} \\
(0.02)\end{array}$ & $\begin{array}{c}-0.198^{\star \star \star} \\
(0.02)\end{array}$ \\
\hline $\begin{array}{l}\text { Nettoeinkommen } \\
\text { in } € / 1000\end{array}$ & & & $\begin{array}{l}0.625^{*} \\
(0.25)\end{array}$ & & & $\begin{array}{l}0.252^{\star *} \\
(0.08)\end{array}$ & $\begin{array}{l}0.269^{\star \star *} \\
(0.08)\end{array}$ & $\begin{array}{l}0.251^{\star *} \\
(0.08)\end{array}$ & $\begin{array}{l}0.250^{* *} \\
(0.08)\end{array}$ & $\begin{array}{l}0.255^{\star *} \\
(0.08)\end{array}$ & $\begin{array}{l}0.113 \\
(0.08)\end{array}$ & $\begin{array}{l}0.141 \\
(0.08)\end{array}$ \\
\hline $\begin{array}{l}\text { Geschlecht } \\
\text { (Ref.: männlich) }\end{array}$ & & & $\begin{array}{l}1.519^{* * *} \\
(0.46)\end{array}$ & & & $\begin{array}{l}1.806^{\star \star *} \\
(0.25)\end{array}$ & $\begin{array}{l}1.844^{\star * *} \\
(0.25)\end{array}$ & $\begin{array}{l}1.806^{\star \star *} \\
(0.25)\end{array}$ & $\begin{array}{l}1.789^{\star \star \star} \\
(0.25)\end{array}$ & $\begin{array}{l}1.819^{\star \star * *} \\
(0.25)\end{array}$ & $\begin{array}{l}1.343^{\star \star *} \\
(0.27)\end{array}$ & $\begin{array}{l}1.390^{\star * *} \\
(0.27)\end{array}$ \\
\hline $\begin{array}{l}\text { Interaktion Frau \& } \\
\text { Nettoeinkommen }\end{array}$ & & & $\begin{array}{c}-0.808^{* *} \\
(0.31)\end{array}$ & & & $\begin{array}{c}-0.791^{\star \star \star} \\
(0.13)\end{array}$ & $\begin{array}{c}-0.787^{\star \star *} \\
(0.13)\end{array}$ & $\begin{array}{c}-0.791^{\star \star \star} \\
(0.13)\end{array}$ & $\begin{array}{c}-0.780^{\star \star \star} \\
(0.13)\end{array}$ & $\begin{array}{c}-0.786^{\star \star *} \\
(0.13)\end{array}$ & $\begin{array}{c}-0.466^{\star *} \\
(0.14)\end{array}$ & $\begin{array}{c}-0.474^{\star *} \\
(0.15)\end{array}$ \\
\hline $\begin{array}{l}\text { Schulabschl. (Ref.: } \\
\text { keine/Hauptschule) }\end{array}$ & & & & & & & & & & & & \\
\hline $\begin{array}{l}\text { Realschulabschluss/ } \\
\text { mittlere Reife }\end{array}$ & & & $\begin{array}{l}0.380 \\
(0.31)\end{array}$ & & & $\begin{array}{r}-0.229 \\
(0.17)\end{array}$ & $\begin{array}{r}-0.197 \\
(0.17)\end{array}$ & $\begin{array}{r}-0.229 \\
(0.17)\end{array}$ & $\begin{array}{r}-0.214 \\
(0.17)\end{array}$ & $\begin{array}{r}-0.215 \\
(0.17)\end{array}$ & $\begin{array}{r}-0.266 \\
(0.18)\end{array}$ & $\begin{array}{r}-0.237 \\
(0.18)\end{array}$ \\
\hline $\begin{array}{l}\text { Abitur / (Fach-) } \\
\text { Hochschulreife }\end{array}$ & & & -0.564 & & & $-0.685^{\star \star \star}$ & $-0.600^{\star \star \star}$ & $-0.683^{\star * *}$ & $-0.653^{\star \star \star}$ & $-0.662^{\star \star \star}$ & $-0.764^{\star \star \star}$ & $-0.686^{\star \star \star}$ \\
\hline Mediatoren & & & $(0.34)$ & & & $(0.17)$ & $(0.17)$ & $(0.17)$ & $(0.17)$ & $(0.17)$ & $(0.18)$ & $(0.19)$ \\
\hline $\begin{array}{l}\text { Großwerden der Kin- } \\
\text { der=größte Freude }\end{array}$ & & & & & & & $\begin{array}{c}0.784^{\star \star \star} \\
(0.13)\end{array}$ & & & & & $\begin{array}{c}0.763^{* * *} \\
(0.15)\end{array}$ \\
\hline Ideale Kinderzahl & & & & & & & & $\begin{array}{c}-0.016 \\
(0.12)\end{array}$ & & & & $\begin{array}{l}0.015 \\
(0.15)\end{array}$ \\
\hline $\begin{array}{l}\text { Negative Beurteilung } \\
\text { Schwangerschaftsab- } \\
\text { bruch }\end{array}$ & & & & & & & & & 0.086 & & & -0.030 \\
\hline & & & & & & & & & $(0.07)$ & & & $(0.07)$ \\
\hline $\begin{array}{l}\text { Traditionelle } \\
\text { Geschlechterrollen }\end{array}$ & & & & & & & & & & 0.095 & & 0.039 \\
\hline $\begin{array}{l}\text { Familienstand } \\
\text { (Ref.: ledig/geschie- } \\
\text { den/getrennt lebend) }\end{array}$ & & & & & & & & & & & & \\
\hline Verheiratet & & & & & & & & & & & $\begin{array}{l}2.018^{* * *} \\
(0.15)\end{array}$ & $\begin{array}{l}2.012^{\star \star \star} \\
(0.15)\end{array}$ \\
\hline Verwitwet & & & & & & & & & & & $\begin{array}{l}1.492^{\star * *} \\
(0.32)\end{array}$ & $\begin{array}{l}1.518^{\star * *} \\
(0.32)\end{array}$ \\
\hline Konstante & $\begin{array}{l}1.313^{* * *} \\
(0.15) \\
\end{array}$ & $\begin{array}{l}1.447^{\star \star \star} \\
(0.25)\end{array}$ & $\begin{array}{l}1.138^{*} \\
(0.49) \\
\end{array}$ & $\begin{array}{l}0.812^{\star \star \star *} \\
(0.06)\end{array}$ & $\begin{array}{r}0.244^{*} \\
(0.11) \\
\end{array}$ & $\begin{array}{l}0.817^{\star \star \star} \\
(0.23)\end{array}$ & $\begin{array}{c}-1.755^{\star \star *} \\
(0.51) \\
\end{array}$ & $\begin{array}{r}0.927^{*} \\
(0.36) \\
\end{array}$ & $\begin{array}{l}0.681^{\star *} \\
(0.25)\end{array}$ & $\begin{array}{l}0.727^{*} \\
(0.30)\end{array}$ & $\begin{array}{c}-0.112 \\
(0.25) \\
\end{array}$ & $\begin{array}{c}-2.639^{\star \star *} \\
(0.58) \\
\end{array}$ \\
\hline $\begin{array}{l}\text { Pseudo- }^{2} \\
\mathrm{~N}\end{array}$ & 0.000 & $\begin{array}{l}0.001 \\
880\end{array}$ & 0.346 & 0.005 & 0.021 & 0.268 & $\begin{array}{l}0.286 \\
1711\end{array}$ & 0.269 & 0.269 & 0.269 & 0.357 & 0.371 \\
\hline
\end{tabular}

Daten: ALLBUS 2012, eigene Berechnungen, ungewichtet. Y=1 zeigt das Vorhandensein wenigstens eines Kindes an. Dargestellt sind die Regressionskoeffizienten und die Standardfehler in Klammern. Nicht dargestellte Variablen: Dummy für fehlende Werte beim Nettoeinkommen sowie den Mediatoren. Pseudo-R2 wurde in einer separaten Schätzung der Truncated-at-zero-Gleichung ermittelt. Signifikanzniveau: $* \mathrm{p}<0.05, * * \mathrm{p}<0.01, * * * \mathrm{p}<0.001$. 
Der positive Regressionskoeffizient in Modell 8.1 zeigt an, dass sich die Wahrscheinlichkeit, Kinder zu haben, in Westdeutschland mit steigender Zentralität erhöht. Der Effekt sinkt allerdings etwas, wenn die sozioökonomischen Drittvariablen kontrolliert werden (Modell 9.1). Die Verringerung des Koeffizienten ist vor allem auf die Kontrolle des Alters zurückzuführen. Wird die westdeutsche Stichprobe auf über 45jährige beschränkt (N=1042), findet sich kein signifikanter Effekt der Zentralität auf Elternschaft mehr. Bei den soziodemographischen Faktoren scheinen sich zusätzlich der Bildungsabschluss und das Geschlecht auszuwirken, da Hauptschulabsolventen und Frauen im ALLBUS seltener kinderlos sind. Zwischen Männern und Frauen ergeben sich beim Effekt von Zentralität auf Elternschaft keine statistisch relevanten Unterschiede (Ergebnisse hier nicht gezeigt).

Doch in welcher Form wirkt sich Zentralität auf die Wahrscheinlichkeit, Kinder zu haben, aus? Wie die Mediationsanalysen (Modelle 10.1 bis 15.1) zeigen, bietet sich vor allem die Institution der Ehe als Erklärung für den Zusammenhang an. Der Einfluss von Zentralität wird deutlich reduziert und ist nicht mehr signifikant, wenn für den Familienstand kontrolliert wird (Modell 14.1 und 15.1). Die Kontrolle der anderen Mediatoren führt nicht zu einer deutlichen Veränderung des Koeffizienten der Zentralität.

\subsection{Zentralität, Kinderzahl und vermittelnde Mechanismen: Multivariate Analysen}

Die Ergebnisse der zweiten Gleichung des Poisson-Logit-Hurdle-Modelle zur Kinderzahl sind in Tabelle 3 dargestellt. Die Vorgehensweise und der Aufbau sind identisch mit Tabelle 2. In Hinblick auf die Kinderzahl bei Eltern zeigt sich zunächst ein ähnlicher Befund wie bei der Wahrscheinlichkeit, überhaupt Kinder zu haben. In den Poisson-Regressionsmodellen haben weder die Konfessionszugehörigkeit noch die Zentralität in den neuen Bundesländern einen Einfluss auf die realisierte Kinderzahl (Modelle 4.2 bis 6.2). ${ }^{9}$ Erwartungsgemäß spielt das Alter eine große Rolle: Ältere Personen haben mehr Kinder. In den alten Bundesländern zeigt sich wiederum sehr deutlich der Einfluss von Zentralität auf die realisierte Kinderanzahl - weitgehend unabhängig von weiteren Variablen im Modell. Je nach Modell steigt die durchschnittliche Kinderzahl um 0.10 bis 0.14 , wenn Zentralität um eine Einheit höher ist (Modelle 8.2 bis 15.2). Konfessionalität hat hingegen keinen Einfluss, sobald die Zentralität kontrolliert wird (Modelle 8.2 bis 15.2). Dies weist darauf hin, dass Zentralität und nicht die bloße Zugehörigkeit zu einer Glaubensgemeinschaft bedeutend für das generative Verhalten ist.

9 Wie in der Logit-Gleichung ist der Unterschied zwischen Ost- und West beim Effekt von Zentralität signifikant (Ergebnisse nicht dargestellt). 
Tabelle 3: Zentralität und Kinderzahl: Mediationsanalyse (Poisson-logit-HurdleRegressionen, 2. Gleichung Truncated-at-zero-Poisson)

\begin{tabular}{|c|c|c|c|c|c|c|c|c|c|c|c|c|}
\hline & \multicolumn{2}{|c|}{ Ostdeutschland } & \multicolumn{4}{|c|}{ Westdeutschland } & \multirow[b]{2}{*}{$\begin{array}{r}\text { Modell } \\
10.2 \\
\end{array}$} & \multirow[b]{2}{*}{$\begin{array}{r}\text { Modell } \\
11.2 \\
\end{array}$} & \multirow[b]{2}{*}{$\begin{array}{r}\text { Modell } \\
12.2 \\
\end{array}$} & \multirow[b]{2}{*}{$\begin{array}{r}\text { Modell } \\
13.2 \\
\end{array}$} & \multirow[b]{2}{*}{$\begin{array}{r}\text { Modell } \\
14.2 \\
\end{array}$} & \multirow[b]{2}{*}{$\begin{array}{r}\text { Modell } \\
15.2 \\
\end{array}$} \\
\hline & $\begin{array}{c}\text { Modell } \\
4.2 \\
\end{array}$ & $\begin{array}{c}\text { Modell } \\
5.2 \\
\end{array}$ & $\begin{array}{r}\text { Modell } \\
6.2 \\
\end{array}$ & \begin{tabular}{|c|} 
Modell \\
7.2 \\
\end{tabular} & $\begin{array}{r}\text { Modell } \\
8.2 \\
\end{array}$ & $\begin{array}{c}\text { Modell } \\
9.2 \\
\end{array}$ & & & & & & \\
\hline Konfessionslosigkeit & $\begin{array}{c}-0.121 \\
(0.07)\end{array}$ & $\begin{array}{c}-0.106 \\
(0.10)\end{array}$ & $\begin{array}{c}-0.073 \\
(0.10)\end{array}$ & $\begin{array}{c}-0.268^{* * *} \\
(0.08)\end{array}$ & $\begin{array}{c}-0.091 \\
(0.09)\end{array}$ & $\begin{array}{c}-0.068 \\
(0.09)\end{array}$ & $\begin{array}{c}-0.064 \\
(0.09)\end{array}$ & $\begin{array}{r}-0.065 \\
(0.09)\end{array}$ & $\begin{array}{c}-0.056 \\
(0.09)\end{array}$ & $\begin{array}{r}-0.065 \\
(0.09)\end{array}$ & $\begin{array}{c}-0.041 \\
(0.09)\end{array}$ & $\begin{array}{r}-0.029 \\
(0.09)\end{array}$ \\
\hline Zentralität & & $\begin{array}{c}0.010 \\
(0.05)\end{array}$ & $\begin{array}{c}0.015 \\
(0.05)\end{array}$ & & $\begin{array}{l}0.143^{\star \star \star} \\
(0.03)\end{array}$ & $\begin{array}{c}0.133^{\star \star *} \\
(0.03)\end{array}$ & $\begin{array}{c}0.130^{* \star \star} \\
(0.03)\end{array}$ & $\begin{array}{c}0.130^{* \star *} \\
(0.03)\end{array}$ & $\begin{array}{c}0.116^{\star \star \star} \\
(0.03)\end{array}$ & $\begin{array}{c}0.128^{\star \star \star} \\
(0.03)\end{array}$ & $\begin{array}{c}0.129^{* \star \star} \\
(0.03)\end{array}$ & $\begin{array}{l}0.108^{\star * *} \\
(0.03)\end{array}$ \\
\hline Alter (in 10 Jahren, zentriert) & & & $\begin{array}{c}0.087^{\star *} \\
(0.03)\end{array}$ & & & $\begin{array}{c}0.083^{* \star *} \\
(0.02)\end{array}$ & $\begin{array}{c}0.085^{\star \star \star} \\
(0.02)\end{array}$ & $\begin{array}{c}0.082^{\star \star \star} \\
(0.02)\end{array}$ & $\begin{array}{c}0.085^{\star \star \star} \\
(0.02)\end{array}$ & $\begin{array}{c}0.080^{* \star \star} \\
(0.02)\end{array}$ & $\begin{array}{c}0.072^{* *} \\
(0.02)\end{array}$ & $\begin{array}{l}0.074^{\star \star} \\
(0.02)\end{array}$ \\
\hline Alter $^{2}$ & & & $\begin{array}{c}-0.053^{\star *} \\
(0.02)\end{array}$ & & & $\begin{array}{c}-0.017 \\
(0.01)\end{array}$ & $\begin{array}{c}-0.018 \\
(0.01)\end{array}$ & $\begin{array}{c}-0.014 \\
(0.01)\end{array}$ & $\begin{array}{c}-0.017 \\
(0.01)\end{array}$ & $\begin{array}{c}-0.019 \\
(0.01)\end{array}$ & $\begin{array}{c}-0.014 \\
(0.01)\end{array}$ & $\begin{array}{c}-0.017 \\
(0.01)\end{array}$ \\
\hline Nettoeinkommen in $€ / 1000$ & & & $\begin{array}{c}0.028 \\
(0.03)\end{array}$ & & & $\begin{array}{c}0.022 \\
(0.03)\end{array}$ & $\begin{array}{c}0.030 \\
(0.03)\end{array}$ & $\begin{array}{c}0.025 \\
(0.03)\end{array}$ & $\begin{array}{l}0.021 \\
(0.03)\end{array}$ & $\begin{array}{c}0.028 \\
(0.03)\end{array}$ & $\begin{array}{c}0.015 \\
(0.03)\end{array}$ & $\begin{array}{l}0.025 \\
(0.03)\end{array}$ \\
\hline Geschlecht (Ref.: männlich) & & & $\begin{array}{c}0.275^{*} \\
(0.12)\end{array}$ & & & $\begin{array}{l}0.126 \\
(0.10)\end{array}$ & $\begin{array}{c}0.140 \\
(0.10)\end{array}$ & $\begin{array}{c}0.113 \\
(0.10)\end{array}$ & $\begin{array}{c}0.110 \\
(0.10)\end{array}$ & $\begin{array}{c}0.141 \\
(0.10)\end{array}$ & $\begin{array}{l}0.090 \\
(0.10)\end{array}$ & $\begin{array}{l}0.104 \\
(0.10)\end{array}$ \\
\hline Interaktion Frau * Einkommen & & & $\begin{array}{c}-0.168 \\
(0.09)\end{array}$ & & & $\begin{array}{c}-0.075 \\
(0.06)\end{array}$ & $\begin{array}{c}-0.083 \\
(0.06)\end{array}$ & $\begin{array}{c}-0.068 \\
(0.06)\end{array}$ & $\begin{array}{c}-0.063 \\
(0.06)\end{array}$ & $\begin{array}{c}-0.075 \\
(0.06)\end{array}$ & $\begin{array}{c}-0.038 \\
(0.06)\end{array}$ & $\begin{array}{c}-0.035 \\
(0.06)\end{array}$ \\
\hline $\begin{array}{l}\text { Schulabschl. (Ref.: } \\
\text { keine/Hauptschule) }\end{array}$ & & & & & & & & & & & & \\
\hline $\begin{array}{l}\text { Realschulabschluss/ } \\
\text { mittlere Reife }\end{array}$ & & & $\begin{array}{r}-0.161 \\
(0.10)\end{array}$ & & & $\begin{array}{r}-0.061 \\
(0.07)\end{array}$ & $\begin{array}{r}-0.063 \\
(0.07)\end{array}$ & $\begin{array}{r}-0.059 \\
(0.07)\end{array}$ & $\begin{array}{r}-0.051 \\
(0.07)\end{array}$ & $\begin{array}{r}-0.059 \\
(0.07)\end{array}$ & $\begin{array}{r}-0.066 \\
(0.07)\end{array}$ & $\begin{array}{r}-0.052 \\
(0.07)\end{array}$ \\
\hline $\begin{array}{l}\text { Abitur / } \\
\text { (Fach-)Hochschulreife }\end{array}$ & & & $\begin{array}{r}-0.131 \\
(0.12)\end{array}$ & & & $\begin{array}{r}-0.042 \\
(0.07)\end{array}$ & $\begin{array}{r}-0.039 \\
(0.07)\end{array}$ & $\begin{array}{r}-0.052 \\
(0.07)\end{array}$ & $\begin{array}{r}-0.023 \\
(0.07)\end{array}$ & $\begin{array}{r}-0.032 \\
(0.07)\end{array}$ & $\begin{array}{r}-0.058 \\
(0.07)\end{array}$ & $\begin{array}{r}-0.027 \\
(0.07)\end{array}$ \\
\hline Mediatoren & & & & & & & & & & & & \\
\hline $\begin{array}{l}\text { Großwerden der Kinder= } \\
\text { größte Freude }\end{array}$ & & & & & & & $\begin{array}{l}0.103 \\
(0.07)\end{array}$ & & & & & $\begin{array}{l}0.087 \\
(0.07)\end{array}$ \\
\hline Ideale Kinderzahl & & & & & & & & $\begin{array}{l}0.103^{\star * \star} \\
(0.03)\end{array}$ & & & & $\begin{array}{l}0.101^{* * *} \\
(0.03)\end{array}$ \\
\hline $\begin{array}{l}\text { Negative Beurteilung Schwan- } \\
\text { gerschaftsabbruch }\end{array}$ & & & & & & & & & $\begin{array}{l}0.065^{*} \\
(0.03)\end{array}$ & & & $\begin{array}{l}0.058^{\star} \\
(0.03)\end{array}$ \\
\hline $\begin{array}{l}\text { Traditionelle Geschlechterrol- } \\
\text { len }\end{array}$ & & & & & & & & & & $\begin{array}{l}0.066 \\
(0.04)\end{array}$ & & $\begin{array}{l}0.055 \\
(0.04)\end{array}$ \\
\hline $\begin{array}{l}\text { Familienstand } \\
\text { (Ref.: ledig/geschie- } \\
\text { den/getrennt lebend) }\end{array}$ & & & & & & & & & & & & \\
\hline Verheiratet & & & & & & & & & & & $\begin{array}{l}0.245^{\star \star} \\
(0.09)\end{array}$ & $\begin{array}{l}0.224^{\star \star} \\
(0.09)\end{array}$ \\
\hline Verwitwet & & & & & & & & & & & $\begin{array}{l}0.191 \\
(0.13)\end{array}$ & $\begin{array}{l}0.169 \\
(0.13)\end{array}$ \\
\hline Konstante & $\begin{array}{l}0.524^{* * *} \\
(0.06)\end{array}$ & $\begin{array}{l}0.505^{* * *} \\
(0.11)\end{array}$ & $\begin{array}{c}0.574^{* * *} \\
(0.15)\end{array}$ & $\begin{array}{l}0.560^{* * *} \\
(0.03)\end{array}$ & $\begin{array}{l}0.267^{\star \star \star} \\
(0.06)\end{array}$ & $\begin{array}{r}0.252^{*} \\
(0.10)\end{array}$ & $\begin{array}{r}-0.164 \\
(0.26)\end{array}$ & $\begin{array}{r}-0.039 \\
(0.13)\end{array}$ & $\begin{array}{l}0.145 \\
(0.11)\end{array}$ & $\begin{array}{l}0.082 \\
(0.13)\end{array}$ & $\begin{array}{l}0.068 \\
(0.12)\end{array}$ & $\begin{array}{r}-0.475 \\
(0.28)\end{array}$ \\
\hline $\begin{array}{l}\text { Pseudo-R2 } \\
\mathrm{N}\end{array}$ & 0.029 & $\begin{array}{l}0.029 \\
880\end{array}$ & 0.041 & 0.025 & 0.035 & 0.042 & $\begin{array}{c}0.043 \\
1711\end{array}$ & 0.046 & 0.043 & 0.043 & 0.044 & 0.048 \\
\hline
\end{tabular}

Daten: ALLBUS 2012, eigene Berechnungen, ungewichtet. Dargestellt sind die Regressions-Koeffizienten und Standardfehler in Klammern. Nicht dargestellte Variablen: Dummy für fehlende Werte beim Nettoeinkommen sowie den Mediatoren. Pseudo-R2 wurde in einer separaten Schätzung der Truncatedat-zero-Gleichung ermittelt. Signifikanzniveau: $* \mathrm{p}<0.05,{ }^{*} \mathrm{p}<0.01, * * * \mathrm{p}<0.001$.

Allerdings gibt es auch bedeutende Unterschiede zu den Analysen zur Elternschaft. Zunächst weisen die Modelle eine sehr geringe Erklärungskraft auf. Wenn der Übergang zur Elternschaft bereits erfolgt ist, lässt sich die realisierte Kinderzahl nur sehr eingeschränkt mit den verwendeten Variablen erklären. Zwar beeinflusst die Zentralität zuverlässig positiv die Kinderzahl, die soziodemografischen Kontrollvariablen haben - mit Ausnahme 
des Alters - jedoch keinen signifikanten Einfluss. Wiederum zeigen sich keine statistisch bedeutsamen Unterschiede zwischen Männern und Frauen beim Effekt von Zentralität.

Die Mediatoren können den Einfluss der Zentralität nicht nennenswert reduzieren. Folglich können die zentralen Mechanismen der Wirkung von Zentralität auf die realisierte Kinderzahl mit den vorliegenden Daten nicht identifiziert werden.

Wird die westdeutsche Stichprobe auf über 45jährige beschränkt ( $N=1042)$, ändert sich der Effekt der Zentralität auf die Kinderzahl nur sehr geringfügig. Dies weist darauf hin, dass es sich nicht um einen Timing-Effekt handelt, sondern religiöse Personen nach Abschluss der Geburtenbiografie durchschnittlich mehr Kinder haben.

\section{Diskussion}

Im vorliegenden Beitrag wurde in einer Sekundäranalyse des ALLBUS 2012 untersucht, ob und warum christlich-religiöse Personen häufiger bzw. mehr Kinder haben als säkulare Personen. Die Befunde zeigen, je ausgeprägter die Religiosität, desto wahrscheinlicher und desto mehr Kinder hat eine Person. Die Zugehörigkeit zu einer christlichen Konfession scheint hingegen keinen von der Religiosität unabhängigen Effekt auf die Fertilität zu haben.

Zudem wurde anhand von Mediationsanalysen untersucht, warum sich Religiosität auf Fertilität auswirkt. Vor allem die Frage, warum sich Religiosität auf die Kinderzahl auswirkt, konnte nicht zufriedenstellend beantwortet werden. Die untersuchten Mechanismen hatten keinen oder nur einen äußerst geringen Einfluss. Allerdings wurden sowohl Religiosität als auch die Mediatoren nach der Geburt der Kinder erhoben, was zu einer Abschwächung bestehender Zusammenhänge geführt haben könnte. Dieses Problem könnte durch die Verwendung von Paneldaten (soweit vorhanden) gelöst werden. Warum religiöse Eltern mehr Kinder haben, kann mit den vorliegenden Querschnittsdaten damit nur teilweise erklärt werden.

Beim Übergang zur Elternschaft sind die Mediationsanalysen hingegen aufschlussreich. Religiosität scheint vor allem über die Heiratsneigung zu wirken. Die Ehe stellt aufgrund der wechselseitigen Verpflichtung zu Solidarität sowie der langfristigen Orientierung eine günstige Rahmenbedingung für die Familiengründung dar. Sowohl in Hinblick auf die Familiengründung als auch -erweiterung hat die Ehe vermutlich auch aufgrund gesetzlicher Privilegien einen positiveren Effekt auf die Fertilität als andere, unverbindlichere Lebensarrangements mit weniger gesetzlich garantierten Absicherungen im Falle einer Trennung. Eine rechtliche Gleichstellung von unehelichen und ehelichen Partnerschaften könnte demnach sowohl die Fertilität in Beziehungen außerhalb der Ehe fördern, als auch die Eheaffinität von Frauen und Männern mit Kinderwunsch reduzieren. Die rechtliche Gleichstellung von Lebensarrangements kann jedoch auch mit einem Verlust an Handlungsoptionen einhergehen, z.B. wenn gesetzliche Rechte und Pflichten auch auf uneheliche Beziehungen angewendet werden und so unverbindliche, langfristige Beziehungen zwischen Erwachsenen kaum mehr möglich sind. ${ }^{10}$

10 Einige Länder haben zur Überbrückung der Kluft zwischen Ehe und ungeregelten Partnerschaften ein weiteres zivilrechtliches Lebensarrangement implementiert. In Frankreich wurde bereits 1999 
Neben den gesetzlichen Privilegien für die Ehe kann die Versorgung mit Betreuungseinrichtungen für Kleinkinder als relevante Rahmenbedingung für das generative Verhalten in den Blick genommen werden. Der Effekt von Zentralität auf Fertilität zeigt sich nur in den alten Bundesländern, wo gleichzeitig die Betreuungsinfrastruktur deutlich schlechter ist und somit die traditionelle innerfamiliäre Arbeitssteilung institutionell begünstigt wird (Bertram 2015; Brose 2006; Bundesinstitut für Bau-, Stadt- und Raumforschung 2016). Aufgrund der Analysen kann die Hypothese formuliert werden, dass religiöse Personen bei einem geringen Betreuungsangebot eher das Risiko einer Elternschaft oder einer größeren Familie eingehen als nicht religiöse. Mit einem umfassenderen Betreuungsangebot für (Klein-)Kinder könnten sich möglicherweise die Fertilitätsmuster säkularer und religiöser Personen auch in Westdeutschland annähern.

Auch wenn die empirischen Befunde darauf hindeuten, dass unabhängig von der Kontrolle sozioökonomischer Drittvariablen ein positiver Zusammenhang zwischen Religiosität und Fertilität besteht, verbleiben offene Fragen und Zweifel.

Aufgrund niedriger Fallzahlen konnten neben Christen keine weiteren Religionsgemeinschaften berücksichtigt oder separat analysiert werden. Mehrere Studien zeigen zwar die Angleichung familialen und demografischen Verhaltens von evangelischen/reformierten und katholischen Christen (eine Übersicht hierzu: Lehrer 2004). Ob Religiosität auch in anderen Religionsgemeinschaften einen vergleichbaren Einfluss auf Fertilität hat, konnte hier jedoch nicht untersucht werden.

Ferner wurden in den Analysen auch Personen ohne abgeschlossene Geburtsbiografie berücksichtigt. Schränkt man die Untersuchungsgruppe auf über 45jährige mit weitgehend abgeschlossener Geburtenbiografie ein, dann findet sich weder bei Frauen noch bei Männern ein signifikanter Zusammenhang zwischen Zentralität und Elternschaft. Dies kann mehrere Ursachen haben, z.B. könnte es sich um einen Timing-Effekt handeln. Demnach dürften religiöse Personen nicht häufiger, sondern nur früher Kinder haben (was allerdings den bisherigen Befunden entgegenstehen würde). Außerdem könnte sich der Zusammenhang von Religiosität und Fertilität im historischen Verlauf verändern und damit den Unterschied zwischen den Kohorten- bzw. Altersgruppen erklären. Und letztlich könnte die Messung der Religiosität in unterschiedlichen Lebensphasen von Bedeutung sein, da ältere Personen tendenziell religiöser sind. Damit ist auch eine Schwachstelle der Analyse angesprochen - die Messungen von Zentralität und generativem Verhalten können weit auseinanderliegen. Die Entscheidung, Personen mit unabgeschlossener Geburtsbiografie zu berücksichtigen, erfolgte somit auch aus dem Grund einer größeren zeitlichen Nähe der Messung von Religiosität und Fertilität sowie der anderenfalls sehr geringen Fallzahlen in den Mediationsanalysen.

Schließlich beinhalten die gezeigten Analysen keine direkte Prüfung der Kausalität immerhin ist es denkbar, dass Elternschaft auch die Religiosität oder den Kindernutzen beeinflusst. Aufgrund nationaler und internationaler Studien gibt es jedoch keinen triftigen Grund, den hier ermittelten Einfluss von Religiosität auf Fertilität zu bezweifeln.

der heute sehr populäre PACS (pact civil de solidarité) eingeführt (Bailly/Rault 2013). Auch in Deutschland, Österreich und der Schweiz gab und gibt es immer wieder entsprechende Vorstöße von Parteien bzw. in der Schweiz aktuell vom Bundesrat. 


\section{Literatur}

Adsera, A. (2006a). Marital fertility and religion in Spain, 1985 and 1999. Population Studies: A Journal of Demography, 60, S. 205-221. doi:10.1080/0032472060068481.

Adsera, A. (2006b). Religion and changes in family-size norms in developed countries. Review of Religious Research, 47, S. 271-286.

Arránz Becker, O., Lois, D. \& Nauck, B. (2010). Unterschiede in den Fertilitätsmustern zwischen ostund westdeutschen Frauen. Differenzierung der Rollen des kulturellen Hintergrunds und des Transformationsprozesses. Zeitschrift für Bevölkerungswissenschaft, 35, S. 35-64. doi:10.4232/10.CPoS-2010-02de.

Bailly, E. \& Rault, W. (2013). Les pacsés en couple hétérosexuel sont-ils différents des mariés? Population \& Sociétés, 497, S. 1-4.

Becher, I. \& El-Menouar, Y. (2014). Geschlechterrollen bei Deutschen und Zuwanderern christlicher und muslimischer Identität. Nürnberg: Bundesamt für Migration und Flüchtlinge (Forschungsbericht 21).

Berghammer, C. \& Schuster, J. (2010). Alles hat seine Stunde? Religiosität und die Zeitpunkte von Ereignissen im Familienverlauf. In: Mazal, W. (Hrsg.), Familie und Religion. Aktuelle Beiträge aus der interdisziplinären Familienforschung. Opladen: Budrich UniPress, S. 11-38.

Berghammer, C. (2012): Church attendance and childbearing: Evidence from a Dutch panel study, 19872005. Population Studies, 66, 2, S. 197-212.

Bertram, H. (2015). Fertilität, Zukunft mit Kindern und die Bedeutung des regionalen Kontextes. In: Jurczyk, K., Lange, A. \& Thiessen, B. (Hrsg), Doing Family: Warum Familienleben heute nicht mehr selbstverständlich ist. Weinheim \& Basel: Beltz Juventa, S. 160-189.

Blume, M., Ramsel, C. \& Graupner, S. (2006). Religiosität als demographischer Faktor - Ein unterschätzter Zusammenhang? Marburg Journal of Religion, 11, S. 1-24.

Brose, N. (2006). Gegen den Strom der Zeit? Vom Einfluss der religiösen Zugehörigkeit und Religiosität auf die Geburt von Kindern und die Wahrnehmung des Kindernutzens. Zeitschrift für Bevölkerungswissenschaften, 31, S. 257-282.

Bund der katholischen Jugend (BDKJ). (2014). Umfrage zu Ehe, Familie und Partnerschaft. www.bdkj. de/bdkjde/themen/vatikan-umfrage/7-zur-offenheit-der-eheleute-fuer-das-leben. html [Stand: 201609-20].

Bundesinstitut für Bau, Stadt- und Raumforschung (2016). Indikatoren und Karten zur Raum- und Stadtentwicklung. www.inkar.de [Stand: 2016-09-20].

Bühner, M. (2011). Einführung in die Test- und Fragebogenkonstruktion. München: Pearson Studium.

Destatis (2012). Geburten in Deutschland 2012. www.destatis.de/DE/Publikationen/Thematisch/ Bevoelkerung/Bevoelkerungsbewegung/BroschuereGeburtenDeutschland0120007129004. pdf?_blob=publicationFile [Stand: 2016-09-20].

Deutsche Bischofskonferenz (DBK) (2014). Die pastoralen Herausforderungen der Familie im Kontext der Evangelisierung. Zusammenfassung der Antworten aus den deutschen (Erz-)Diözesen auf die Fragen im Vorbereitungsdokument für die III. Außerordentliche Vollversammlung der Bischofssynode 2014.

www.dbk.de/fileadmin/redaktion/diverse_downloads/presse_2014/2014-012a-Fragebogen-Diepastoralen-Herausforderungen-der-Familie.pdf [Stand: 2016-09-20].

Diel, C. \& König, M. (2011). Religiosität und Geschlechtergleichheit - Ein Vergleich türkischer Immigranten mit der deutschen Mehrheitsbevölkerung. In: Meyer, H. \& Schubert, K., Politik und Islam. Wiesbaden: Springer VS, 191-215.

Dinno, A. (2009). Implementing Horn's parallel analysis for principal component analysis and factor analysis. The Stata Journal, 9, S. 291-298.

Ehrhardt, J., Huinink, J., Kohli, M. \& Staudinger, U. M. (2012). Theorien der Fertilität. In: Stock, G., Bertram, H. \& Fürnkranz-Prskawetz, A. (Hrsg.), Zukunft mit Kindern. Frankfurt am Main: Campus Verlag, S. 72-115. 
Evangelische Kirche in Deutschland (EKD) (2004). Die Rolle der Frau in der EKD. www.ekd.de/bevollmaechtigter/stellungnahmen/52400.html [Stand: 2016-09-20].

Evangelische Kirche in Deutschland (EKD) (2014). Engagement und Indifferenz. Kirchengemeinschaft und soziale Praxis. V. EKD-Erhebung über Kirchenmitgliedschaft. www.ekd.de/download/ekd_v_kmu2014.pdf [Stand 2016-09-20].

Esser, H. (2002). In guten wie in schlechten Tagen? Das Framing der Ehe und das Risiko der Scheidung. Eine Anwendung und ein Test des Modells der Frame-Selektion. Kölner Zeitschrift für Soziologie und Sozialpsychologie, 54, S. 27-63. doi:10.1007/ s11577-002-0002-4.

Fowid (Forschungsgruppe Weltanschauungen in Deutschland (2005). Schwangerschaftsabbruch, Religionszugehörigkeit,1982-2000.

www.fowid.de/fileadmin/datenarchiv/Schwangerschaftsabbruch_Religionszugehoerig_1982_2000. pdf [Stand: 2016-09-20].

Frejka, T. \& Westhoff, C. F. (2006). Religion, religiousness and fertility in the U.S. and in Europe. Rostock: Max-Planck-Institut für demografische Forschung MPIDR Working Paper 2006, 13). www.demogr.mpg.de/papers/working/wp-2006-013.pdf [Stand 2016-09-20].

Fuchs, S. (2009). Religion und Demographie - Erkenntnisse aus der empirischen Sozialforschung. Die Neue Ordnung, 63, S. 348-361.

Fuchs, S. (2014). Gesellschaft ohne Kinder. Woran die neue Familienpolitik scheitert. Wiesbaden: Springer VS. doi:10.1007/978-3-658-03390-3_1.

Guske, K. (2014). Zwischen Bibel und Grundgesetz. Die Religionspolitik der Evangelikalen in Deutschland. Wiesbaden: Springer VS. doi:10.1007/978-3-658-03847-2 1.

Hayford, S. R. \& Morgan S. P. (2008). Religiosity and fertility in the United States: The role of fertility intentions. Social Forces, 86, S. 1163-1188. doi:10.1353/ sof.0.0000.

Heineck, G. (2006). The relationship between religion and fertility - Evidence from Austria. Papers on Economics of Religion, 06, S. 1-26.

Hilbe, J. M. (2014). Modeling count data. Cambridge: Cambridge University Press. doi: $10.1017 /$ CBO9781139236065.

Huber, S. (2003). Zentralität und Inhalt. Ein neues multidimensionales Messmodell von Religiosität. Opladen: Leske + Budrich. doi:10.1007/978-3-663-11908-1.

Huber, S. (2008). Kerndimensionen, Zentralität und Inhalt. Ein interdisziplinäres Modell der Religiosität. Journal für Psychologie, 16, S. 1-17.

Huber, S. \& Huber, O. W. (2012). The Centrality of Religiosity Scale (CRS). Religions, 3, S. 710-724. doi: $10.3390 /$ rel3030710.

Hubert, S. (2010). Fördert Religion die Fertilität? Eine empirische Untersuchung anhand des pairfamDatensatzes. Familienprisma, 4, S.18-21.

Hubert, S. (2015). The Impact of religiosity on fertility. A comparative analysis of France, Hungary, Norway, and Germany. Wiesbaden: Springer VS. doi:10.1007/978-3-658-07008-3.

Katechismus der katholischen Kirche (KKK). (1997). www.vatican.va/archive/DEU0035/_INDEX.HTM [Stand: 2016-09-20].

Kraft, K. \& Neimann, S. (2009). Impact of educational and religious homogamy on marital stability. Bonn: Institute for the Study of Labor (Discussion Paper 4491).

Kreyenfeld, M. (2003). Crisis or adaptation - Reconsidered: A comparison of East and West German fertility patterns in the first six years after the „Wende“. European Journal of Population, 19, S. 303-329. doi:10.1023/A:1024992712815.

Kröhnert, S. \& Klingholz, R. (2010). Glaube, Macht und Kinder. Erobern religiöse Menschen mit vielen Nachkommen die Welt? Berlin: Berlin-Institut für Bevölkerung und Entwicklung.

Lehrer, E. L. (2004). Religion as a determinant of economic and demographic behavior in the United States. Population and Development Review, 30, S. 707-726. doi:10.1111/j.1728-4457.2004.00038.x.

Lois, D. (2008). Einflüsse von Lebensstilmerkmalen auf den Übergang in die erste Ehe. Zeitschrift für Familienforschung, 20, S. 11-33. 
Lois, D. (2011a). Kirchenmitgliedschaft und Kirchgangshäufigkeit im Zeitverlauf - Eine Trendanalyse unter Berücksichtigung von Ost-West-Unterschieden. Zeitschrift für Bevölkerungswissenschaft, 36, S. $127-160$.

Lois, D. (2011b). Wie verändert sich Religiosität im Lebenslauf. Eine Panelanalyse unter Berücksichtigung von Ost-West-Unterschieden. Kölner Zeitschrift für Soziologie und Sozialpsychologie, 63, S. 83-110. doi:10.1007/s11577-010-0124-z.

Mc Quillan, K. (2004). When does religion influence fertility? Population and Development Review, 30, S. 25-56.

Müller, A. (2006). Die sexuelle Sozialisation in der weiblichen Adoleszenz. Mädchen und jungen Frauen deutscher und türkischer Herkunft im Vergleich. Münster: Waxmann.

Nauck, B. (2001). Der Wert von Kindern für ihre Eltern. „Value of Children“ als spezielle Handlungstheorie generativen Verhaltens und von Generationsbeziehungen im interkulturellen Vergleich. Kölner Zeitschrift für Soziologie und Sozialpsychologie, 53, S. 407-435. doi:10.1007/s11577-001-0073-7.

Philipov, D. \& Berghammer, C. (2007). Religion and fertility ideals, intentions and behaviour - A comparative study of European countries. Vienna Yearbook of Population Research, 5, S. 271-305. doi: 10.1553/populationyearbook2007s271.

Pollack, D. \& Müller, O. (2013). Religionsmonitor 2013. Religiosität und Zusammenhalt in Deutschland. Gütersloh: Bertelsmann Stiftung.

Ramsel, C. (2011). Deutschland und seine Kinder - eine Frage der Religiosität. Gesellschaftliche und individuelle Bedingungen der Reproduktion religiöser Menschen. https://publikationen.uni-tuebingen.de/xmlui/handle/10900/46920 [Stand: 2016-09-20].

Roloff, J. (2004). Mögliches Verhalten von Frauen in West- und Ostdeutschland bei einer ungewollten Schwangerschaft und die Akzeptanz des Schwangerschaftsabbruchs - ein Zeitvergleich. Wiesbaden: Bundesinstitut für Bevölkerungsforschung (Materialien zur Bevölkerungswissenschaft, 113).

Sandmann, T. (2015): Religiosität und Fertilität. Marburg: Philipps-Universität Marburg (M.A.-Abschlussarbeit).

Srikanthan, A. \& Reid, R. L. (2008). Religious and cultural influences on contraception. Journal of Obstetrics and Gynaecology Canada/Journal d'obstétrique et de gynécologie du Canada, 30, S. 129137. doi:10.1016/S1701-2163(16)32736-0.

Wagner, M. \& Weiß, B. (2003). Bilanz der deutschen Scheidungsforschung. Versuch einer MetaAnalyse. Zeitschrift für Soziologie, 32, S. 29-39. doi:10.1515/zfsoz-2003-0102.

Wenzel, S. (2010). Konvergenz oder Divergenz? Einstellungen zur Erwerbstätigkeit von Müttern in Ostund Westdeutschland. Gender, 3, S. 59-76.

Eingereicht am/Submitted am: 03.11.2016

Angenommen am/Accepted on: 13.10.2017

Autoren/Authors:

Tim Sandmann $\dagger$

zuletzt: Institut Empirica, CVJM-Hochschule, Kassel

PD Dr. Klaus Preisner

Universität Zürich

Institut für Soziologie

Andreasstrasse 15

8050 Zürich

Schweiz/Switzerland

E-Mail/Email: preisner@soziologie.uzh.ch 